\title{
Cognitive Strategies Dependent on the Hippocampus and Caudate Nucleus in Human Navigation: Variability and Change with Practice
}

\author{
Giuseppe Iaria, ${ }^{1,2,3}$ Michael Petrides, ${ }^{2}$ Alain Dagher, ${ }^{2}$ Bruce Pike, ${ }^{2}$ and Véronique D. Bohbot ${ }^{1}$ \\ ${ }^{1}$ Douglas Hospital Research Center, McGill University, Verdun, Quebec, Canada, H4H 1R3, ${ }^{2}$ Montreal Neurological Institute, McGill University, Montreal, \\ Quebec, Canada H3A 2B4, 3Dipartimento di Psicologia, Università di Roma "La Sapienza", Roma, Italy, and Instituto di Ricovero e Cura a Carattere \\ Scientifico Fondazione Santa Lucia, Roma, Italy 00179
}

The human brain activity related to strategies for navigating in space and how it changes with practice was investigated with functional magnetic resonance imaging. Subjects used two different strategies to solve a place-learning task in a computer-generated virtual environment. One-half of the subjects used spatial landmarks to navigate in the early phase of training, and these subjects showed increased activation of the right hippocampus. The other half used a nonspatial strategy and showed, with practice, sustained increased activity within the caudate nucleus during navigation. Activation common to both groups was observed in the posterior parietal and frontal cortex. These results provide the first evidence for spontaneous variability and shift in neural mechanisms during navigation in humans.

Key words: spatial memory; place learning; striatum; virtual environment; topographical amnesia; basal ganglia

\section{Introduction}

Different strategies can be used to navigate in the environment (Berthoz, 2001). For instance, to reach a target location, one can use the cognitive map of the environment (spatial memory) by thinking about the landmarks and their spatial relationships (O'Keefe and Nadel, 1978). Alternatively, one can use distance from a single landmark as a reference or make choices with respect to body motion, independent of the landmarks available in the environment. These different strategies probably depend on, to some extent, practice in navigating and may rely on different parts of the brain.

In rats, place learning involves two different memory systems subserved by the hippocampus and the striatum (caudate nucleus and putamen), respectively (O'Keefe and Nadel, 1978; McDonald and White, 1994, 1995; Packard and McGaugh, 1996; White and McDonald, 2002). In the early phase of learning, the hippocampus is involved in the rapid acquisition of spatial information, allowing rats to reach a target from any starting position (O'Keefe and Nadel, 1978). The striatum is involved in a slower learning process (Packard and McGaugh, 1996) that relies on

Received Sept. 17, 2002; revised April 30, 2003; accepted May 2, 2003.

This study was supported by Natural Sciences and Engineering Research Council of Canada Operating Grant 239920 and start-up funds from Douglas Hospital Research Centre to V.B. This study was also supported in part by a Canadian Institutes of Health Research operating grant to A.D., B.P., and M.P. We thank Alex Zijdenbos for help with Unreal, Mike Ferreira and Pierre Ahad for help in adapting the behavioral task to the fMRI protocol, Keith Worsley and Serge Dumoulin for valuable help in the analysis, and Martin LePage and Kate Watkins for helpful comments on a previous version of this manuscript.

Correspondence should be addressed to Dr. Veronique Bohbot, Department of Psychiatry, McGill University, Douglas Hospital Research Center, Frank B. Common Building, 6875 LaSalle Boulevard, Verdun, Quebec, Canada, H4H 1R3. E-mail: veronique.bohbot@mcgill.ca.

Copyright $\odot 2003$ Society for Neuroscience $\quad$ 0270-6474/03/235945-08\$15.00/0 rewarded stimulus-response (S-R) behavior (Packard and Knowlton, 2002; White and McDonald, 2002), i.e., gradually learning particular body turns in response to stimuli, which allow the animal to reach a target location from one starting position (Eichenbaum et al., 1990). The use of the striatal system increases with practice in navigating in the environment (Packard and McGaugh, 1996). Thus, rats can reach a target place by relying on the contribution of the hippocampal or the striatal neural systems (McDonald and White, 1994), depending on whether the animal is in an early or late phase of training (Packard and McGaugh, 1996).

Studies of human subjects with temporal lobe resections, including the hippocampus (Goldstein et al., 1989; Feigenbaum et al., 1996; Maguire et al., 1996; Morris et al., 1996; Abrahams et al., 1997), or selective damage to the hippocampus and the parahippocampal cortex (Bohbot et al., 1998; Holdstock et al., 2000) suggest that these brain regions play a critical role in spatial memory. Furthermore, functional neuroimaging studies (Aguirre et al., 1996; Maguire et al., 1998; Mellet et al., 2000) have shown activation of the medial temporal lobe related to the spatial representation of the virtual environment in which the subject is navigating. However, no studies have been reported showing the modulation of brain activity while humans spontaneously adopt different navigational strategies in a place-learning task, and as these strategies change with practice. This was the aim of the present study. Experiment 1 investigated the natural variability in how human subjects navigate in a virtual environment. Experiment 2 used functional magnetic resonance imaging (fMRI) to map the neural systems involved in solving the task using different strategies and the changes in the pattern of brain activity with practice. 


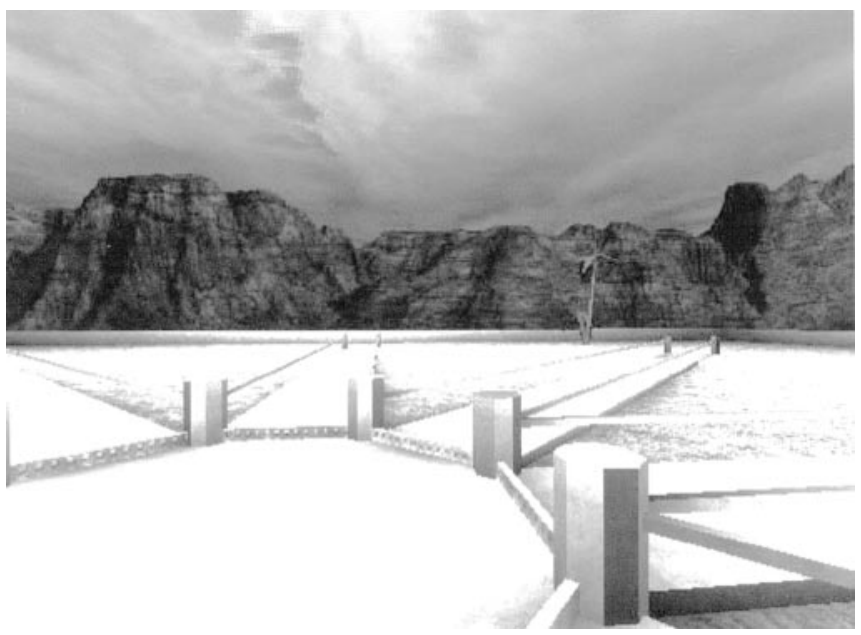

Figure 1. A view of the virtual environment. Note that the landscape and a tree can be viewed at a distance, whereas the objects down the stairs at the end of the arms are not visible from the center of the maze.

\section{Materials and Methods}

Experiment 1: behavioral study

Subjects. Fifty normal right-handed subjects ( 25 males and 25 females matched in age; mean age, $27.7 \pm 4.7$ years) were tested. None had a history of neurological disorders. Informed consent was obtained in a manner approved by the local ethics committee.

Task. A commercially available computer game (Unreal; Epic Games, Raleigh, NC) was used to create a virtual environment and administer the virtual task on a computer screen. The virtual environment was composed of an eight-arm radial maze with a central starting location. The maze was surrounded by a landscape (mountains and sunset), two trees, and a short wall located between the landscape and the trees (Fig. 1). At the end of each arm, there was a staircase leading to the location where, in some of the arms, an object could be picked up. Therefore, there were no objects or cues that could indicate the location of the target objects from the center of the maze. The subjects used a keypad with forward, backward, left turn, and right turn buttons to move within the environment. Before testing, the subjects spent a few minutes moving in a virtual room that was different from the experimental environment to practice the motor aspects of the task. When the subjects were comfortable using the keypad, the experimenter gave the instructions, and the experiment started.

Subjects always started a trial from the center of the radial maze. There were three types of trials, all of which were composed of two parts. In Part 1 , four of the eight arms were accessible with objects at the end of each arm; in Part 2, all arms were accessible and objects were present in the four arms that had been blocked in Part 1. The subjects were told to retrieve all four objects from the accessible arms in Part 1 and remember which arms they visited to avoid them in Part 2. An error consisted of an entry into an arm that did not contain an object. In trial type A (sequence A), in Part 1, arms 1, 3, 4, and 6 were accessible and contained an object; in Part 2, the four objects were located at the end of the four previously blocked arms (i.e., arms 2, 5, 7 and 8). In trial type B (sequence B), a different sequence of accessible arms was used. In Part 1, arms 2, 3, 7, and 8 were accessible, and in Part 2, the objects were located at the end of arms $1,4,5$, and 6 . Trial type $\mathrm{C}$ was a probe trial. In Part 1 , this trial was identical to the trial type A (sequence A). In Part 2, however, the walls around the radial maze were raised to conceal the landscape, and the trees were removed so that no landmarks were visible. Also, eight objects were present (one at the end of each arm). For this and every trial, subjects finished the trial after four objects had been picked up. The following was the rationale of the probe trial: if subjects were using a spatial strategy in which the landmarks present in the environment were relevant to perform the task, this change in the environment should result in an increase in errors. In contrast, if subjects were using a nonspatial strategy, no increase in errors should occur. Testing was divided into four consecu- tive sections composed of 4,5,5, and 4 trials, respectively. In section I, the subjects performed the following order of trials: trial types A, B, A, and C. In the second and third sections, which were considered the "training phase," the subjects performed only trial type A. Section IV was identical to section I (i.e., trial types A, B, A, C).

At the end of the experiment, the subjects were debriefed. They were asked to report how they solved the task from the beginning to the end of the experiment. Subjects were categorized as using a nonspatial strategy when they associated the arms with numbers or letters, or they counted the arms (clockwise or counterclockwise) from a single starting point. If they used at least two landmarks and did not mention a nonspatial strategy, they were categorized as using spatial memory. Subjects who mentioned using several landmarks at the beginning and later shifted to counting were placed into the "shift group." If the subjects did not mention the start position, they were asked if they remembered whether the starting position was the same or different at every trial.

Two experimenters independently evaluated the reports of the subjects and assigned the subjects to a particular strategy group depending on the method used to navigate in the environment. The independent judgments of the experimenters were correlated to evaluate their consistency. We measured the errors the subjects made during the test and the time spent to perform the tasks in each section.

Note that the use of the term place in this study, is similar to the term place used by White and McDonald (2002) and Eichenbaum et al. (1990), which refers to a location that can be reached in either of two ways: by learning its relationship to environmental landmarks that surround it, or by acquiring a series of reinforced responses from a unique starting point. The term spatial specifically refers to the use of an array of environmental landmarks to perform the place-learning task as defined by O'Keefe and Nadel (1978). The virtual maze task was intentionally designed to allow two distinct place-learning strategies, and, therefore, it is not a purely spatial task (O'Keefe and Nadel, 1978).

\section{Experiment 2: fMRI study}

Subjects. Fourteen young healthy subjects (mean age, 25.3; SD, 2.8; seven males) participated in this study. The subjects were right-handed and had no history of neurological disorders. Informed consent was obtained in a manner approved by the local ethics committee.

Task. The experimental task and the virtual environment were identical to those used in Experiment 1. However, in the fMRI study, there was an additional visuo-motor control condition during which the subjects were asked to pick up the same objects randomly placed at the end of four arms. This time, the objects in the visuo-motor control condition were visible from the center of the maze. In Part 1 of the experimental trials, four of the eight arms were accessible with objects at the end of each arm that were not visible from the center of the maze. In Part 2, all arms were accessible and four objects were present in the four arms that were blocked in Part 1. The subjects were asked to retrieve all four objects from the accessible arms in Part 1 and remember which arms were visited to avoid these and find the four objects in Part 2. As in Experiment 1, there were three trial types (A, B, and C). Because of time constraints, fewer trials were administered in the fMRI task compared with the behavioral task. The following order of trials was performed by the subjects: A, B, C, A, A, A, B, C. There were eight scans (otherwise called runs) of $7 \mathrm{~min}$ each. In each scan, the subjects performed one experimental trial and several visuo-motor control trials, linked to one another until the end of the 7 min scan. Before scanning, as in Experiment 1, the subjects spent a few minutes moving in a virtual room that was different from the experimental environment to practice the motor aspects of the task. At the end of the experiment, the subjects were debriefed using the same procedure adopted in Experiment 1. We recorded all of the errors as well as the time the subjects spent performing the experimental trials.

fMRI acquisition data. The scanning session consisted of eight scans (7 min each). At the very beginning of each scan, before the experimental and visuo-motor control trials, the subjects performed a task identical to the visuo-motor control with the exception that there was one visible object instead of four. This allowed us to control for equilibration effects by excluding the first few frames of each scan from the analysis. Because of the variability between subjects in the time taken to perform the tasks, 
we used homemade software to record frame times; every keystroke made by the subject as well as the keystrokes by the experimenter indicated transition from one task to another. This allowed us to exclude from the analysis the frames acquired during the translations between the tasks. The MRI scans were obtained with a Siemens Vision $1.5 \mathrm{~T}$ system (Siemens AG, Erlangen, Germany). For the anatomical images, a threedimensional gradient echo acquisition was used to collect 80 contiguous $2 \mathrm{~mm}$ T1-weighted images in the sagittal plane. The functional scanning session began with a sagittal localizer, followed by a series of test blood oxygenation level-dependent (BOLD) scans. Each functional scan was acquired using 26 contiguous $5 \mathrm{~mm}$ axial slices positioned parallel to the hippocampus and covering the entire brain $[64 \times 64$ matrix; echo time (TE), 50 msec; number of frames, 105; time between measurements, 4 sec; field of view, $320 \mathrm{~mm}$ ]. BOLD signal images were spatially smoothed (6 mm Gaussian kernel), corrected for motion, and linearly transformed into standard stereotaxic space (Talairach and Tournoux, 1988) using in-house software (Collins et al., 1994). Individual $t$ maps of the comparisons between experimental and control tasks in each scan, as well as group-averaged statistical images and correlation maps, were obtained using the FMRISTAT software package (Worsley et al., 2002). The $t$-statistic thresholds corrected for multiple comparisons for the whole brain volume were $t=4.43(p<0.05)$, and $t=5.25(p<0.001)$. For the predicted searches, the corrected thresholds were determined to be $t=$ $3.25(p<0.05)$, and $t=4.30(p<0.001)$, on the basis of the sum of the volumes of the right hippocampus and right caudate nucleus $(3500+$ $5500 \mathrm{~mm}^{3}$, respectively). For the correlation analyses, the uncorrected threshold for the predicted searches (in the hippocampus and caudate nucleus) was $t=1.96(p<0.05)$. The threshold corrected for multiple comparisons for the whole brain volume $(t=4.43 ; p<0.05)$ was used for other brain areas for the correlation analyses.

\section{Results}

\section{Experiment 1: behavioral study}

The debriefing reports indicated that at the beginning of the session, 23 of 50 subjects solved the task using spatial memory (i.e., they used the relationships between landmarks present in the environment), and 27 of 50 subjects solved the task using a nonspatial strategy (i.e., they counted the arms clockwise or counterclockwise from the start position or a single landmark). By the end of the test, $36(72 \%)$ subjects were using the nonspatial strategy, and only 14 (28\%) subjects were using spatial memory. Thus, with practice, some subjects shifted from using spatial memory to the nonspatial strategy (i.e., they first used environmental landmarks to orient themselves and later counted the arms from a single starting point). On the basis of the verbal reports of the subjects, two experimenters independently assigned the subjects to the different groups (spatial memory, shift, nonspatial strategy) with $96 \%$ overlap. If we assign subjects who made errors during the high-wall probe trials to the spatial memory group, and those who made no errors to the nonspatial strategy group, there was a $68 \%$ overlap on the first probe trial with the classification on the basis of the verbal reports and $78 \%$ overlap on the second. The following are examples of subjects' reports. (1) Spatial memory group: "I used the trees and the sun. In the first two trials, I also used the mountains. After that, I continued to use the trees and the sun. I do not remember if it was always the same starting position, because I only paid attention to the environment." (2) Shift group: "I started using the mountains and the trees. After I made errors, I decided to change strategy. So, I counted the arms counterclockwise. Afterwards, I realized that the starting position was always the same, I always counted the arms from that point." (3) Nonspatial strategy group: "I always counted the arms from the tree. In the first high-wall trial, I guessed the first arm and then I used the same sequence I used before. In the second one, I used the starting position, which I realized was always the same."

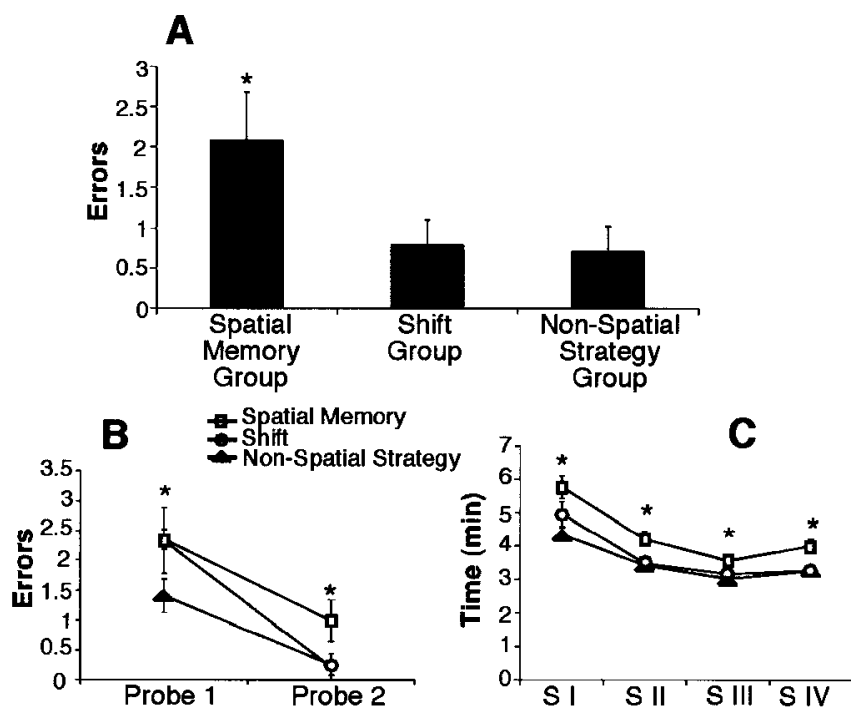

Figure 2. The behavioral results. $A$, The total number of errors made in the training phase (sections II and III) averaged across subjects in the spatial memory, shift, and nonspatial strategy groups. $B$, The number of errors made while performing the probe trial in sections I (probe 1) and IV (probe 2) averaged across subjects in the spatial memory, shift, and nonspatial strategy groups. C, The average time that the spatial memory, shift, and nonspatial strategy groups required to perform one trial in sections (S) I to IV of the experiment. SEM are shown. Asterisks indicate that the spatial memory group is different from the nonspatial strategy group; $p<0.05$.

Thus, the place-learning test that we administered can be solved using two different strategies: one relying on the landmarks present in the environment (spatial memory), and the other relying on counting the arms from a constant start position or single landmark, ignoring the relationship between the elements present in the environment (nonspatial strategy). Thus, subjects spontaneously adopted one strategy or the other, and, in some cases, spontaneously shifted from spatial memory to a nonspatial strategy.

\section{Errors}

We analyzed the errors for the three groups during the first and second probe trials. The ANOVA group (spatial memory, shift, nonspatial strategy) by probe (first, second), with the number of errors as repeated measures, revealed significant main effects of group $[F(2,47)=3.625 ; p<0.05]$ and probe $[F(1,47)=50.962$; $p<0.001]$. The main effect of group showed that the subjects who used spatial memory (spatial memory group) made more errors than the subjects who used the nonspatial strategy (nonspatial strategy group) $(p<0.05)$. The main effect of probe showed that all subjects made more errors in the first probe than the second probe $(p<0.001)$. In the first $(t(38)=2.87 ; p<0.01)$ and second $(t(39)=2.09 ; p<0.05)$ probe trials, the spatial memory group made more errors than the nonspatial strategy group. As expected, there were no differences between the shift and the spatial memory groups in the first probe $(t(10)=0.04$; $p>0.05$; nonsignificant) and between the shift and the nonspatial group on the second probe $(t(34)=0.11 ; p>0.05$; nonsignificant) (Fig. $2 B$ ).

We then analyzed the errors for the three groups (spatial memory, shift, nonspatial strategy) during the training phase (sections II and III together). $t$ test analyses revealed that the spatial memory group made more errors than the nonspatial strategy group $(t(34)=2.24 ; p<0.05)$; the difference between the spatial memory and shift groups approached statistical significance $(t(19)=1.90 ; p=0.07)$. There was no difference between 
the shift and nonspatial memory groups $(t(34)=0.13 ; p>0.05)$ (Fig. 2A).

\section{Time}

Figure $2 C$ shows the time spent during the different sections of the experiment. An ANOVA group (spatial memory, nonspatial strategy, shift) by section (I, II, III, IV) (with minutes as repeated measures) showed significant main effects of the group $[F(2,47)=9.41 ; p<0.0005]$ and section $[F(3,47)=92.06 ; p<$ $0.0001]$. The main effect of group showed that the subjects who used spatial memory (spatial memory group) took longer to perform the task than the shift group $(p<0.05)$ and those who used the nonspatial strategy (nonspatial strategy group) $(p<0.001)$. The main effect of section showed that all subjects performed the task progressively faster, confirming that they improved on the task $(p<0.001)$.

\section{Sex}

We analyzed the gender of the subjects with respect to the strategy used to solve the task. There was no difference between the number of men and women in the different groups on the basis of the strategy used to perform the test. There were no differences in the errors made by men and women $(t(39)=0.39 ; p>0.05$; nonsignificant). However, we found a gender effect on the time to perform sections I to IV: males being faster than females $[t(48)=$ 2.48; $p<0.05]$.

In summary, on the basis of the verbal reports, subjects were classified into three groups: spatial memory, nonspatial strategy, and shift groups. The subjects were assigned to the same groups by two experimenters with a $96 \%$ overlap. The groups dissociated themselves in terms of errors made on the probe trials, errors made throughout training, and latencies to perform the task. The verbal statements for a given group were clearly different from the other and reflected the navigational approach used. For these reasons, we planned on using verbal statements to group subjects in our fMRI experiment.

\section{Experiment 2: fMRI study}

\section{Behavioral data}

At the end of the scanning sessions, the subjects were debriefed. On the basis of their reports, we found that seven subjects (mean age, 24.4; SD, 2.9) (four males, three females) solved the task from the beginning to the end of the experiment by counting the arms (nonspatial strategy group). The other seven subjects (mean age, 26.1; SD, 2.8) (three males, four females) solved the task, first by using the relationship between landmarks present in the environment (spatial memory group), and after some practice, they shifted to using the nonspatial strategy (i.e., counting the arms). Note that this group corresponds to the shift group of the behavioral study.

We did not find statistical differences between the two groups in terms of errors made on the experimental tasks [repeated measures ANOVA; $F(1,7)=0.97 ; p=0.327$; nsec]. Moreover, in the probe trials (scans 3 and 8), the two groups did not differ in error rates. This finding suggests that by scan 3 , the spatial memory group had begun switching to the nonspatial strategy.

\section{fMRI data}

First, we examined the brain regions involved in the performance of the task for the entire subject pool $(n=14)$. We found statistically increased BOLD signal during the performance of the task compared with the visuo-motor control bilaterally in the posterior parietal cortex (area 7), the putamen, the right caudate nu-

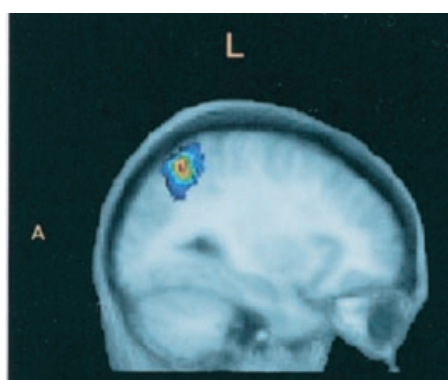

$X=-28$

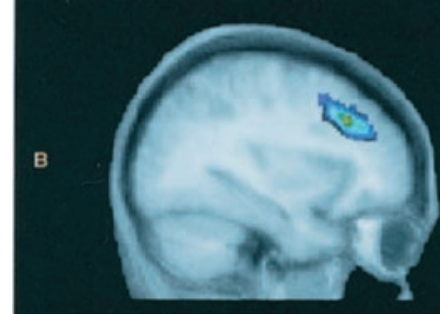

$x=-32$

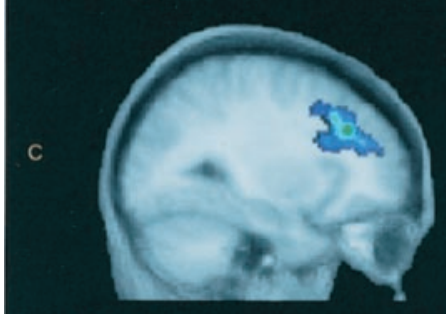

$X=-28$

$=24$

$x=18$

$x=30$
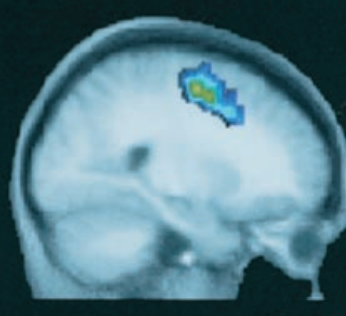
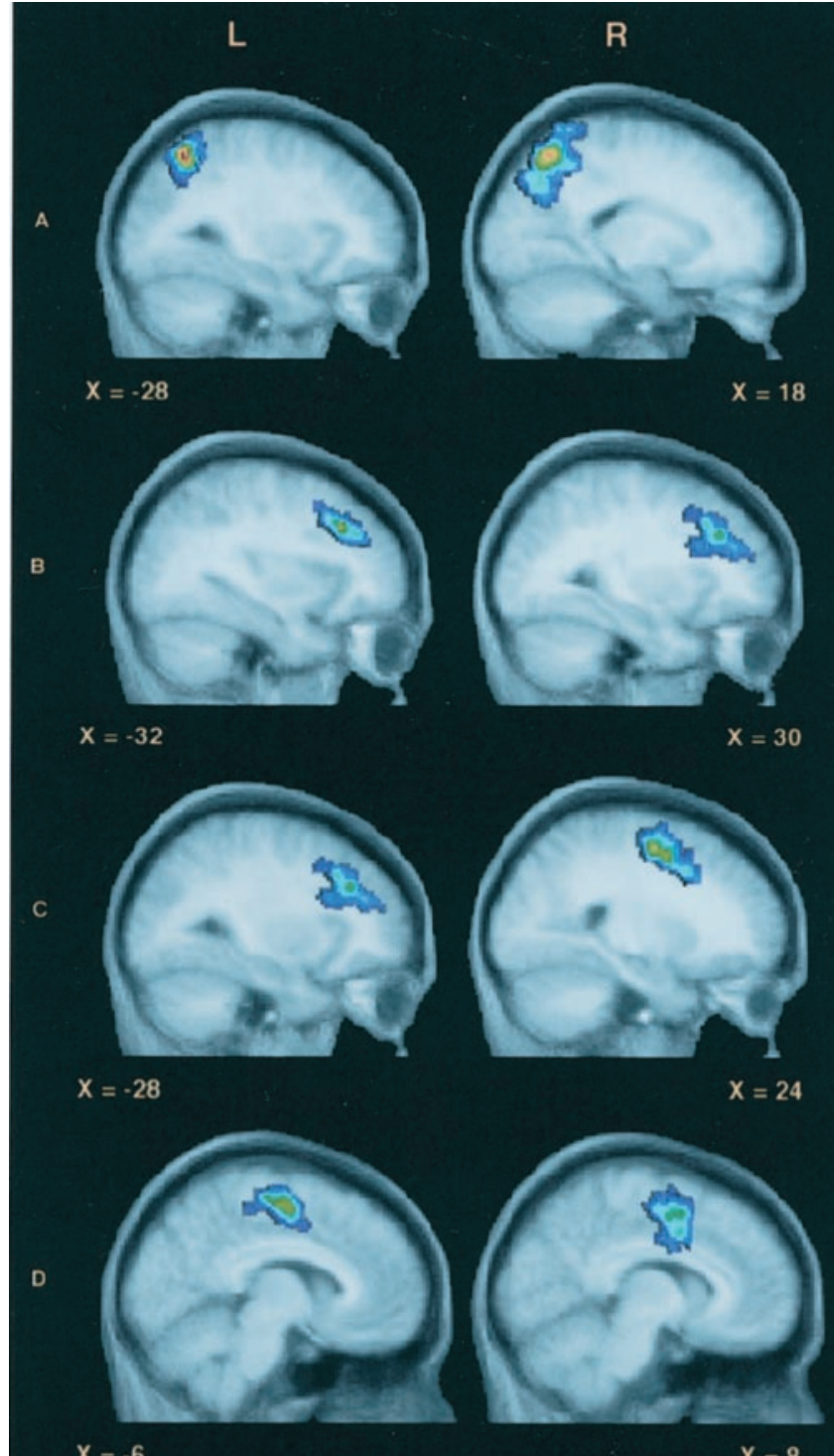

$x=-6$

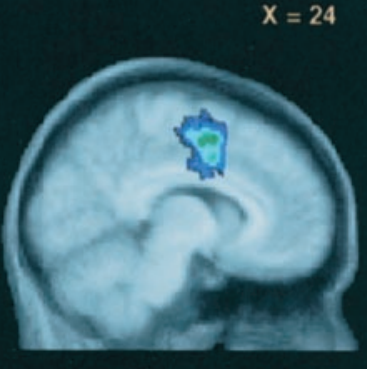

$X=8$

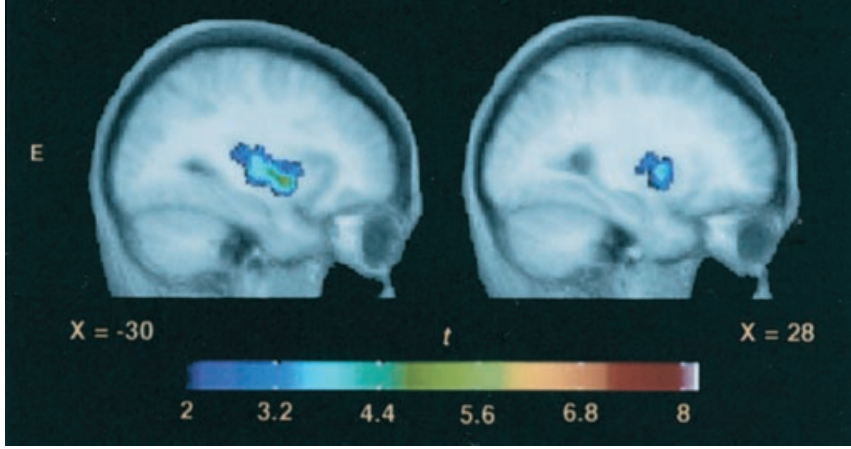

Figure 3. Brain activity common to both spatial memory and nonspatial strategy groups (experimental minus control task). The $t$ maps are superimposed onto the anatomical average of all participants and displayed in the sagittal plane. A, Posterior parietal cortex. B, Middorsolateral prefrontal cortex. C, Motor-premotor cortical region. $D$, Supplementary motor cortex. E, Putamen. L, Left hemisphere; $R$, right hemisphere.

cleus, the left middle occipital gyrus, and the right cerebellum. In addition, there was bilateral activation of the mid-dorsolateral prefrontal cortex (area 9 of 46), primary motor cortex (area 4), and the supplementary motor cortex (area 6), extending into the adjacent right cingulate motor region (Fig. 3). Table 1 reports the 
Table 1. Brain activity common to both spatial memory and nonspatial strategy groups

\begin{tabular}{lrrrl}
\hline & \multicolumn{3}{l}{ Talairach coordinates } & \\
\cline { 2 - 3 } Anatomical region & $x$ & $y$ & $z$ & $t$ value \\
\hline Right & 18 & -64 & 54 & 6.5 \\
$\quad$ Parietal cortex & 30 & 34 & 28 & 4.9 \\
$\quad$ Middle frontal gyrus & 24 & -12 & 54 & 5.76 \\
$\quad$ Motor-premotor cortical region & 4 & 4 & 48 & 4.51 \\
& 8 & -4 & 48 & 4.94 \\
Supplementary motor cortex & 12 & 18 & 40 & 4.75 \\
$\quad$ Cingulate cortex & 12 & 4 & 40 & 4.77 \\
& 28 & 4 & 4 & 4.05 \\
Putamen & 16 & -8 & 22 & 4.07 \\
Caudate nucleus & 30 & -34 & -42 & 5.02 \\
$\quad$ Cerebellum & & & & \\
Left & -28 & -60 & 56 & 7.16 \\
$\quad$ Parietal cortex & -32 & 26 & 28 & 5.31 \\
$\quad$ Middle frontal gyrus & -28 & -8 & 52 & 5.79 \\
Motor-premotor cortical region & -32 & -24 & 62 & 5.76 \\
$\quad$ Supplementary motor cortex & -6 & -10 & 54 & 5.02 \\
$\quad$ Middle occipital gyrus & -32 & -82 & 16 & 4.6 \\
$\quad$ Putamen & -30 & 4 & 0 & 4.99 \\
\hline
\end{tabular}

$t$ values and stereotaxic coordinates (Talairach and Tournoux, 1988) of the voxels of peak activation.

We then analyzed the fMRI data of the spatial memory group $(n=7)$ separately from that of the nonspatial strategy group $(n=$ 7) to investigate our hypothesis that the hippocampus and caudate nucleus would be differentially involved depending on the navigational method. The experimental and probe conditions were contrasted with the control condition performed in every scan.

In the spatial memory group, there was significantly greater BOLD signal in the experimental as compared with the control condition in the right hippocampus in the first (Fig. 4A) and second scans (Table 2). In contrast, the nonspatial strategy group showed no activity increase in the hippocampus in any of the scans, but it demonstrated significant activity in the caudate nucleus (Fig. $4 B$ ) in scans $4,5,6$, and 8 (Table 2). The increase in caudate nucleus activity in the seventh $\operatorname{scan}(x=10 ; y=-4 ; z=$ $20 ; t=2.94)$ approached statistical significance. Thus, with practice, activity in the caudate nucleus emerged in this group and was sustained until the end of the experiment (i.e., scans 4, 5, 6, 7, and $8)$. In the spatial memory group, activity in the caudate nucleus was inconsistent, appearing in only scans 2 and 8 . It is worth noting that the pattern of rewarded arms in the experimental condition of scans 2 and 7 (trial type B) were different from the standard pattern that was present on all other trials (trial type A and C). The difference in hippocampal activation between the two groups in scan 1 was statistically significant in a direct comparison when the activity of the nonspatial strategy group was subtracted from the activity of the spatial memory group (Fig. 4A).

A similar pattern of fMRI results was obtained when subjects were reclassified into the two groups using both verbal reports and the errors made in the first probe trial. The five subjects who made $\geq 1$ error when the environmental landmarks were removed were assigned to the spatial memory group, whereas the five subjects who did not make any errors when the landmarks were removed were assigned to the nonspatial strategy group. The remaining four subjects were ambiguous, because their reports did not correspond to the errors made on probe trials. For example, subjects who said they ignored the multiple landmarks

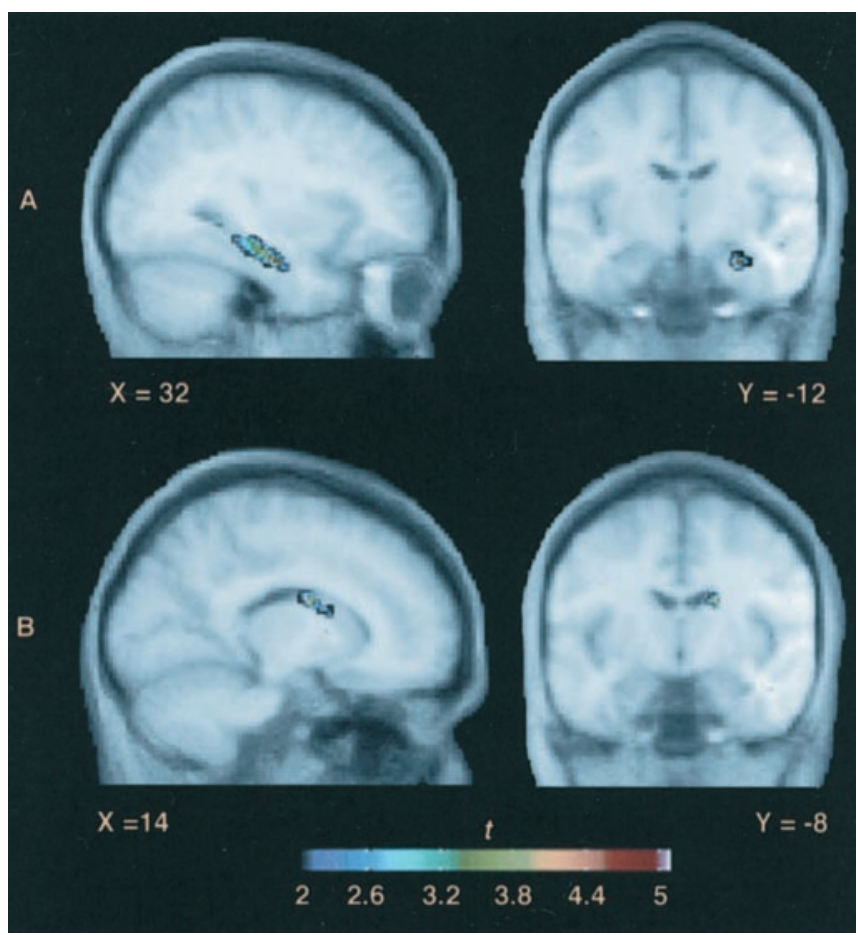

Figure 4. Activity in the hippocampus and caudate nucleus found in the spatial memory group and nonspatial strategy group, respectively. The $t$ maps are superimposed onto the anatomical average of all participants and displayed in the sagittal and coronal planes. $A$, Activity in the right hippocampus when contrasting the experimental and control conditions of the spatial memory group, minus those of the nonspatial strategy group in the first scan ( $x=$ $32 ; y=-14 ; z=-20 ; t=4.41)$. $B$, Activity in the right caudate nucleus found in the nonspatial strategy group ( $\operatorname{scan} 5)(x=14 ; y=-8 ; z=22 ; t=4.04)$.

in the environment and counted from a single landmark, such as a tree, could have made errors on probe trials when landmarks were absent. Consequently, ambiguous cases were removed from the analysis. When the two groups were formed using both the verbal reports and errors on the first probe, the correspondence with the original classification on the basis of the verbal reports alone was five of seven subjects in each group (71\%). As in the previous analysis, a peak of activity in the right hippocampus was observed in the first scan of the spatial memory group only $(x=$ $32 ; y=-14 ; z=-20 ; t=3.94)$. No significant activity was found in the hippocampus of the nonspatial strategy group. Instead, sustained activity was found in the caudate nucleus of the nonspatial strategy group on scans $3(x=8 ; y=14 ; z=12 ; t=3.38)$, $4(x=18 ; y=-8 ; z=26 ; t=3.75), 6(x=16 ; y=-18 ; z=24$; $t=3.52)$, and $8(x=10 ; y=-4 ; z=20 ; t=5.59)$. This also contrasts with the lower level of activity of the caudate nucleus in the spatial memory group ( $\operatorname{scan} 2, x=12, y=-8, z=20, t=$ 3.56; $\operatorname{scan} 3, x=-16, y=4, z=22, t=4.04$ ). In summary, taking errors on probe trials as well as verbal reports into account to group subjects yielded a similar pattern of activation as the grouping on the basis of the verbal reports only.

To explore in greater depth the relationship between brain activity and performance in both spatial memory and nonspatial strategy groups, we correlated the increase in BOLD signal with the number of errors and the latency during the experimental task across all scans. The results showed that in the spatial memory group, the number of errors was negatively correlated with BOLD signal increases in the right caudate nucleus and positively correlated with BOLD signal increase in the hippocampus bilaterally (Table 3). In contrast, in the nonspatial strategy group, the 
Table 2. Brain activity found in the hippocampus and caudate nucleus of the spatial memory and nonspatial strategy groups

\begin{tabular}{lcccc}
\hline & \multicolumn{2}{l}{ Talairach coordinates } & \\
\cline { 2 - 4 } Anatomical region & $x$ & $y$ & $z$ & \\
\hline Spatial memory group & & & & \\
\hline $\begin{array}{l}\text { Right hippocampus } \\
\quad \text { scan 1 }\end{array}$ & 32 & -14 & -20 & 4.41 \\
$\quad$ scan 2 & 22 & -16 & -14 & 3.49 \\
Right caudate nucleus & 14 & -6 & 20 & 4.82 \\
$\quad$ scan 2 & 24 & -22 & 24 & 4.65 \\
$\quad$ scan 8 & & & & \\
Left caudate nucleus & -20 & -2 & 24 & 3.58 \\
$\quad$ scan 8 & & & & \\
Nonspatial strategy group & & & & \\
\hline Right caudate nucleus & 20 & 8 & 18 & 3.81 \\
$\quad$ scan 4 & 14 & -8 & 22 & 4.04 \\
$\quad$ scan 5 & 20 & -24 & 22 & 4.41 \\
$\quad$ scan 6 & 8 & -4 & 20 & 5.36 \\
$\quad$ scan 8 & & & & \\
Left caudate nucleus & -12 & -10 & 18 & 4.3 \\
$\quad$ scan 6 & & & & \\
\hline
\end{tabular}

Table 3. Correlation between the BOLD signal increases and error rate in the spatial memory and nonspatial strategy groups

\begin{tabular}{lrrrr}
\hline & \multicolumn{2}{c}{ Talairach coordinates } & \\
\cline { 2 - 3 } Anatomical region & $x$ & $y$ & $z$ & \\
\hline Spatial memory group (errors) & & & & \\
\hline $\begin{array}{l}\text { Positive correlation } \\
\quad \text { Right hippocampus }\end{array}$ & 32 & -14 & -20 & 2.38 \\
$\quad$ Left hippocampus & -34 & -36 & -8 & 2.86 \\
Negative correlation & & & & \\
$\quad$ Right caudate nucleus & 14 & -6 & 18 & -3.75 \\
Nonspatial strategy group (errors) & & & & \\
\hline Positive correlation & & & & \\
$\quad$ Right angular gyrus (area 39) & 52 & -70 & 24 & 4.48 \\
Negative correlation & & & & \\
$\quad$ Right caudate nucleus & 14 & -16 & 22 & -3.12 \\
$\quad$ Left caudate nucleus & -18 & -14 & 24 & -3.91 \\
$\quad$ Left parietal cortex (area 7) & -26 & -60 & 56 & -4.85 \\
$\quad$ Right cerebellum & 0 & -58 & -26 & -9.65 \\
$\quad$ Left cerebellum & -6 & -62 & -22 & -10.32 \\
\hline
\end{tabular}

number of errors negatively correlated with BOLD signal increase in the left parietal cortex (area 7), caudate nucleus, and cerebellum. There was a positive correlation between BOLD signal increases and error rate in the right angular gyrus (area 39). Similar correlations were observed with latency in both groups (Table 4). Thus, the hippocampus was more active in the spatial memory group when they made more errors and took longer to perform the task, whereas the caudate nucleus was more active with better performance in both groups.

\section{Discussion}

This study investigated the changes in brain activity while human subjects spontaneously adopted different strategies to navigation, and how these were modified with practice. Experiment 1 showed that $46 \%$ of subjects used spatial memory by relying on the relationship between landmarks in the environment, and the others counted the arms and ignored the array of environmental landmarks. Importantly, we found that the subjects spontaneously adopted one of the two strategies. With practice, $39 \%$ of the subjects who initially used spatial memory later shifted to the
Table 4. Correlation between the BOLD signal increases and latency in the spatial memory and nonspatial strategy groups

\begin{tabular}{|c|c|c|c|c|}
\hline \multirow[b]{2}{*}{ Anatomical region } & \multicolumn{3}{|c|}{ Talairach coordinates } & \multirow[b]{2}{*}{$t$ value } \\
\hline & $x$ & $y$ & $z$ & \\
\hline \multicolumn{5}{|l|}{ Spatial memory group (latency) } \\
\hline \multicolumn{5}{|l|}{ Positive correlation } \\
\hline Right hippocampus & 32 & -34 & -8 & 2.53 \\
\hline Left hippocampus & -26 & -34 & -6 & 2.32 \\
\hline Negative correlation & & & & \\
\hline Right superior temporal gyrus (area 39) & 58 & -60 & 36 & -5.93 \\
\hline Right cingulate gyrus (area 32) & 10 & 20 & 30 & -5.08 \\
\hline Right caudate nucleus & 10 & 10 & -4 & -3.43 \\
\hline \multicolumn{5}{|l|}{ Nonspatial strategy group (latency) } \\
\hline \multicolumn{5}{|l|}{ Negative correlation } \\
\hline Right caudate nucleus & 14 & -18 & 24 & -3.84 \\
\hline Left caudate nucleus & -18 & -16 & 24 & -4.03 \\
\hline Right cerebellum & 0 & -58 & -26 & -9.71 \\
\hline Left cerebellum & -6 & -62 & -22 & -10.36 \\
\hline
\end{tabular}

nonspatial strategy, whereas no subject shifted from the nonspatial strategy to spatial memory. These practice-related changes in the strategy, used in the place-learning task, are consistent with previous results in normal rats (Packard and McGaugh, 1996). The probe trials support the debriefing reports, in that there was a statistically significant larger number of errors in the spatial memory group relative to the nonspatial strategy group when the environmental landmarks were removed. These behavioral results suggest a natural variability in the strategies adopted by human subjects faced with a navigation task. This natural variability needs to be taken into account in studies that investigate the neural basis of human navigation, because the strategy adopted by a subject is likely to influence the resulting cognitive processes and, therefore, the imaging results and task performance. These behavioral findings were the basis of Experiment 2, in which fMRI was used to test the hypothesis that the hippocampus and caudate nucleus would be differently involved during the performance of a place-learning task, depending on the strategy used to navigate in the environment. We also hypothesized that the activation pattern would change with practice (i.e., as subjects in the spatial group changed strategy, the activation in the hippocampus would disappear and activity in the caudate nucleus would emerge).

The pattern of brain activation common to all subjects, regardless of the strategy taken in solving the virtual maze task, is consistent with previous functional imaging studies of navigation (Aguirre et al., 1996; Maguire et al., 1998; Mellet et al., 2000). Compared with the visuo-motor control task, performance of the virtual maze task resulted in increased activity within the posterior parietal cortex, consistent with a critical role in spatial perception and movement in space known from lesion studies in humans (Mesulam, 1981; Posner et al., 1984) and monkeys (Petrides and Iversen, 1979) and functional neuroimaging studies (Ungerleider and Haxby, 1994). The posterior parietal cortex in the primate brain has been shown to project to the parahippocampal cortex (Van Hoesen, 1982; Suzuki, 1996), which is also involved in navigation (Aguirre et al., 1996). There was also increased activity in the motor-premotor cortical region and the supplementary motor cortex, which are anatomically closely linked with the posterior parietal cortex (Petrides and Pandya, 1984). There is considerable evidence from neurophysiological studies that this posterior parietal to premotor and supplemen- 
tary motor circuit is involved in the higher level control of movement in space (Andersen and Gnadt, 1989; Milner and Goodale, 1995). Another common area of increased activation in both groups of subjects during the performance of the virtual maze task was the mid-dorsolateral prefrontal cortex (area 9 of 46). Increased activation in this region was expected, because it has been consistently shown to be involved in tasks that require subjects to monitor their response choices, whether the choices to be monitored are spatial or not (Petrides, 1996). In the present task, successful performance requires that, in addition to navigation, the subjects keep track of the arms that have been visited versus the arms that still need to be visited.

The examination of activity patterns, specific to the two strategies used by the subjects in solving the present task, showed increased activity during the performance of the task in the right hippocampus only in the group of subjects who were using spatial memory. Importantly, a contrast between the experimental and visuo-motor control trials of the spatial memory group, minus those of the nonspatial group, revealed an activation of the right hippocampus on trial 1 (Fig. 4). This finding is consistent with previous imaging (Maguire et al., 1998) and neuropsychological (Bohbot et al., 1998) studies, providing strong evidence that the hippocampus is critically involved when the cognitive strategy requires spatial memory (i.e., the use of a cognitive map of the environment). In sharp contrast, the group that adopted the nonspatial strategy did not show hippocampal activity but a sustained increase in BOLD signal in the caudate nucleus in the later stages of task performance compared with control.

The present results are consistent with previous animal data (McDonald and White, 1994, 1995; Packard and McGaugh, 1996). However, the comparison between the nonspatial strategy adopted by humans and the S-R behavior described in rats deserves additional consideration. The analogy lies in the fact that the nonspatial group makes a series of S-R associations. To obtain the objects from arms 7, 8,2, and 5, subjects who counted from the starting position would make a response of going forward to enter the arm ahead ( $\operatorname{arm} 7$ ), make a response to the first left on exiting $\operatorname{arm} 7$ ( $\operatorname{arm} 8$ ), and then take the second left ( $\operatorname{arm} 2)$ and third left ( $\operatorname{arm} 5)$. It is reasonable to assume that the repetition of these $\mathrm{S}-\mathrm{R}$ associations leads to habitual responses. There is evidence that this mechanism involves the striatum or caudate nucleus in both rats and humans. The fact that a decrease in activation of the caudate nucleus was observed with the change in pattern of rewarded arms (trial 7) supports this hypothesis. Our results are also consistent with an fMRI study by Poldrack et al. (2001), in which a declarative and nondeclarative classification learning task was used to show that the medial temporal lobe is involved early in learning, whereas the caudate nucleus is involved in a later phase when subjects make faster classification responses. These results are in accord with our observation that, in the spatial memory group, hippocampal activity was seen only during the early phase of task performance (scan 1 and 2). Importantly, with practice, subjects who used a nonspatial strategy showed activity of the caudate nucleus, which appeared at the later stage of task performance (scan 4) and remained present until the end. This suggests that the caudate nucleus is constantly involved when subjects use a procedural approach to task performance, which is associated with rapid habitual responses (Packard and Knowlton, 2002).

To test the hypothesis that involvement of the caudate nucleus, rather than the hippocampus, is associated with improved performance on this task, we correlated the BOLD signal with accuracy and latency in performance in both groups separately.
We found that the BOLD signal increase in the hippocampus was correlated with poor task performance only in the spatial memory group (the greater the number of errors and the longer time needed to perform the task, the greater the BOLD increase in the hippocampus). This result is consistent with our behavioral finding in which the spatial memory group made more errors and took longer to perform the test, confirming that, in this task, performance that relies on spatial memory is less efficient. However, the positive correlation between the fMRI signal and errors or between the fMRI signal and latencies in the spatial memory group does show that the hippocampus is most active during the learning process in the spatial memory group (i.e., while they were making errors). It is therefore of interest that no subject shifted from a nonspatial strategy to spatial memory, whereas several subjects who were initially using the spatial memory later shifted to the nonspatial strategy. In contrast, BOLD signal increase in the caudate nucleus was found to correlate with improved performance in both groups of subjects, supporting once again the behavioral data in which both groups showed improved performance with practice. The role of the caudate nucleus performing in an automatic manner may suggest adaptive mechanisms in which the human brain optimizes responses in performing familiar behavior. Previous studies have shown the critical role of the caudate nucleus in performing familiar tasks and adapting fast responses (Poldrack et al., 2001). Here, we suggest that the same phenomenon occurs in human navigation. These results are in accord with animal (McDonald and White, 1994) and human (Maguire et al., 1998) studies, suggesting that the hippocampal and striatal systems play different roles in navigation.

In summary, the present study provides evidence that human subjects spontaneously adopt different strategies to solve a navigation task and these strategies lead to differential activity in the hippocampus and caudate nucleus. The hippocampus is only involved in the early phase of performance when spatial memory is used. Because practice leads to the development of a habitual approach to the task, the caudate nucleus becomes involved in a sustained manner. The habitual approach is more efficient and associated with activation in the caudate nucleus. These findings provide evidence of a shift in neural mechanism of the human brain are consistent and extend previous work conducted in rodents (Packard and McGaugh, 1996; White and McDonald, 2002).

\section{References}

Abrahams S, Pickering A, Polkey CE, Morris RG (1997) Spatial memory deficits in patients with unilateral damage to the right hippocampal formation. Neuropsychologia 35:11-24.

Aguirre GK, Detre JA, Alsop DC, D’Esposito M (1996) The parahippocampus subserves topographical learning in man. Cereb Cortex 6:823-829.

Andersen R, Gnadt JW (1989) Role of posterior parietal cortex in saccadic eye movements. In: The neurobiology of saccadic eye movement (Wurtz RH, Goldberg ME, eds), pp 315-335. Amsterdam: Elsevier.

Berthoz A (2001) Neural basis of spatial orientation and memory of routes: topokinetic memory or topokinesthesic memory. Rev Neurol (Paris) 157:779-789.

Bohbot VD, Kalina M, Stepankova K, Spackova N, Petrides M, Nadel L (1998) Spatial memory deficits in patients with lesions to the right hippocampus and to the right parahippocampal cortex. Neuropsychologia 36:1217-1238.

Collins DL, Neelin P, Peters TM, Evans AC (1994) Automatic 3D intersubject registration of MR volumetric data in standardized Talairach space. J Comput Assist Tomogr 18:192-205.

Eichenbaum H, Stewart C, Morris RG (1990) Hippocampal representation in place learning. J Neurosci 10:3531-3542.

Feigenbaum JD, Polkey CE, Morris RG (1996) Deficits in spatial working 
memory after unilateral temporal lobectomy in man. Neuropsychologia 34:163-176.

Goldstein LH, Canavan AG, Polkey CE (1989) Cognitive mapping after unilateral temporal lobectomy. Neuropsychologia 27:167-177.

Holdstock JS, Mayes AR, Cezayirli E, Isaac CL, Aggleton JP, Roberts N (2000) A comparison of egocentric and allocentric spatial memory in a patient with selective hippocampal damage. Neuropsychologia 38:410-425.

Maguire EA, Burke T, Phillips J, Staunton H (1996) Topographical disorientation following unilateral temporal lobe lesions in humans. Neuropsychologia 34:993-1001.

Maguire EA, Burgess N, Donnett JG, Frackowiak RS, Frith CD, O’Keefe J (1998) Knowing where and getting there: a human navigation network. Science 280:921-924.

McDonald RJ, White NM (1994) Parallel information processing in the water maze: evidence for independent memory systems involving dorsal striatum and hippocampus. Behav Neural Biol 61:260-270.

McDonald RJ, White NM (1995) Hippocampal and nonhippocampal contributions to place learning in rats. Behav Neurosci 109:579-593.

Mellet E, Briscogne S, Tzourio-Mazoyer N, Ghaem O, Petit L, Zago L, Etard O, Berthoz A, Mazoyer B, Denis M (2000) Neural correlates of topographic mental exploration: the impact of route versus survey perspective learning. NeuroImage 12:588-600.

Mesulam MM (1981) A cortical network for directed attention and unilateral neglect. Ann Neurol 10:309-325.

Milner AD, Goodale MA (1995) The visual brain in action. New York: Oxford UP.

Morris RG, Pickering A, Abrahams S, Feigenbaum JD (1996) Space and the hippocampal formation in humans. Brain Res Bull 40:487-490.

O’Keefe J, Nadel L (1978) The hippocampus as a cognitive map. Oxford: Clarendon.

Packard MG, Knowlton BJ (2002) Learning and memory functions of the basal ganglia. Annu Rev Neurosci 25:563-593.
Packard MG, McGaugh JL (1996) Inactivation of hippocampus or caudate nucleus with lidocaine differentially affects expression of place and response learning. Neurobiol Learn Mem 65:65-72.

Petrides M (1996) Specialized systems for the processing of mnemonic information within the primate frontal cortex. Philos Trans R Soc Lond B Biol Sci 351:1455-1461; discussion 1461-1452.

Petrides M, Iversen SD (1979) Restricted posterior parietal lesions in the rhesus monkey and performance on visuospatial tasks. Brain Res 161:63-77.

Petrides M, Pandya DN (1984) Projections to the frontal cortex from the posterior parietal region in the rhesus monkey. J Comp Neurol 228:105-116.

Poldrack RA, Clark J, Pare-Blagoev EJ, Shohamy D, Creso Moyano J, Myers C, Gluck MA (2001) Interactive memory systems in the human brain. Nature 414:546-550.

Posner MI, Walker JA, Friedrich FJ, Rafal RD (1984) Effects of parietal injury on covert orienting of attention. J Neurosci 4:1863-1874.

Suzuki WA (1996) Neuranatomy of the monkey enthorinal, perirhinal and parahippocampal cortices: organization of cortical inputs and interconnection with amygdala and striatum. Semin Neurosci 8:3-12.

Talairach J, Tournoux P (1988) Co-planar stereotaxic atlas of the human brain. New York: Thieme.

Ungerleider LG, Haxby JV (1994) "What" and "where" in the human brain. Curr Opin Neurobiol 4:157-165.

Van Hoesen GW (1982) The parahippocampal gyrus. Trends Neurosci 5:345-350.

White NM, McDonald RJ (2002) Multiple parallel memory systems in the brain of the rat. Neurobiol Learn Mem 77:125-184.

Worsley KJ, Liao CH, Aston J, Petre V, Duncan GH, Morales F, Evans AC (2002) A general statistical analysis for fMRI data. NeuroImage 15:1-15. 